\title{
Application of Things: A Step beyond Web of Things
}

\author{
J.A. Ortega Ramírez ${ }^{1}$, L.M. Soria Morillo ${ }^{1}$, Alexander Kröner ${ }^{2}$, \\ and J.A. Álvarez García ${ }^{1}$ \\ ${ }^{1}$ University of Seville, Seville, Spain \\ \{jortega, 1soria, jaalvarez\}@us.es \\ ${ }^{2}$ German Research Center for Artificial Intelligence, Saarbrucken, Germany \\ alexander.kroener@dfki.de
}

\begin{abstract}
Internet of Things has achieved a great expectation in the last few years, largely due to possibilities in the field of the interaction between user and environment. This interaction allows users to get involved in their environments, managing at all times each item that surrounds them. Specially, because of complex services delivered by surrounding item, increasingly it is necessary to help users to choose what services are offered and what are theirs functionalities. Is this paper, is presented a new approach to the human-items interaction by using the concept of Internet of Things. In this way, the user can not only run the different applications offers by smart-things, but to share theirs results through many social networks and allows the remote execution of services provided by the own user and his/her social environment. Furthermore, a structure will be defined to assure the best user comfort when information sharing between the device and the environments was needed.
\end{abstract}

Keywords: RFID tag, Information sharing, Web of Things, Mobile environments, Pervasive computing.

\section{Introduction}

\subsection{Motivation}

Since in 1999 the concept of Internet of Things born from the hands of Roy Want (W. Roy, 1999), many things have changed. First, the society has suffered a technological revolution and everyone interact in their daily lives with electronic devices like mobile phones, electronic agendas, personal computers and so on. The number of functionalities that these elements give to people is huge and sometimes, can be reached to overload the environments. The first sequel of this fact is that services potentials are not exploited by users and in most cases the functionalities used are the simplest ones. This problem occurs often with elderly people or with non-technological users.

Internet of Things can help to these users by providing a way to shows the available services that every smart-thing surrounding them can offer. Obviously, when we refer to smart-things not only make reference to electronic devices, but every object that can be identified by using the technology. In this field lies the potential of IoT, due to in most of related works are used RFID or NFC (Near Field Communication) 
tags to identify the objects (Kranz, Holleis, \& Schmidt, 2010) (Alexander Kröner, 2008). RFID and NFC are technologies that allow writing in a magnetic tag some information so that later, that information will be able to be read by using many sensors. However, these technologies have an important drawback: the scope. RFID technology using passive tags, i.e. tags that don't need any external power supply, only can be read from a few centimeters. Although, recently a RFID reader has been developed by the Daily RFID company, which can be read passive tags from 15 meters. In this case, the main problem of the reader is its size, upper than $40 \mathrm{~cm}$.

However, this technology has a crucial advantage over other identification methods: the cost. We will focus on the embedded RFID and NFC readers due to in the future, studying the market trends; most generation mobile phones could include this technology. This is possible thanks to the increase in electronic payment (Ondrus, 2007) (Lacmanovic, Radulovic, \& Lacmanovic, 2010) and, as mentioned above, the reduced cost of both, readers and tags.

Internet of Things philosophy is based on that every object must have an electronic resource which al-lows identifying it. This resource must be composed by two elements: an identification method (in our case RFID or NFC technology) and an information provider. The last one is often a server with internet connection through which are offered all services to clients.

In this work we will develop a new way to present on the mobile phone services that smart-things give to users and interact with their environment. In this case, all the information needed by these services will be stored in the own mobile phone, being the charge of managing the user information and becoming in his/her Digital Me (Andreas Björklind, 2003). So, Digital Object Memory project (Boris Brandherm, 2010), in which objects have information about the user and his/her interaction with them, might improve its QoS (especially in terms of personalization) based on data from the mobile phone.

One step further, the proposed work will receive a feedback from Object Memories, with the aim of improve the QoS of local services.

Furthermore, this proposal not only allows sharing information between user's device and smart-thing, but the user can share all information obtained from the items with her/his friends, due to the system allows connections with social networks for this purpose. Additionally, the user can share some smart services with friends, and getting others to use services offered by these smart-things.

Due to the system make use of personal information, this must take in mind the aim of assure the information privacy. This will be made by use a core-application around which will be executed services and functions from smart-things. Thus, the user can be sure that only the needed information will be shared with the smart-thing and it's more, the own user will be able to determine what information wants to share and what not.

All these features will make the user can use the functionality provided for his/her environment in a more natural way and without interact directly with the smart-thing. Therefore the user must not learn how the item can be used, but only will have to watch the phone screen and execute the service. 


\subsection{Contributions of This Work}

The novelty of this work relies on users must be execute and share services offered by smart-things that surround them, and this execution must be based on a minimum user interaction. To this end, we introduce in this paper a set of facilities and techniques that will make possible achieve the cited aim:

- Control of shared information. Mobile devices store much personal information about the user. Possibilities of share this information are huge, but must be present that an improper access to it could be fatal. For this reason, in this paper will be exposed a method to notify the user what in-formation is requested by each service. Further-more, security clearances will be stored in the own device, so the next time the user interacts with this service, the information will be shared automatically.

- Sharing services functionality. Users don't only can execute a service provided by some smart-thing, but also they will be able to share this service with any trusted people. This will be made by using social networks as sharing platform and delivering to their members the service address. As mentioned above, each service will have a concrete http address, so a call to this service by using the correct parameters will execute the functionality. For this purpose, has been developed a method to determine if some service is or not shareable and if a person has privileges to execute it.

- On-line and Off-line services. The proposed sys-tem has been made for allowing users select if must be executed the on-line or off-line version of some service. As we will see later, some service could be executed using a user-interface application or an automatic execution by web-services calls. The first one can be seen as a traditional application in which users must interact to for obtain a result. On the other hand, using on-line methods, the system delivers to users an autonomous process to send the information requested by some service and execute it to obtain results.

- Identification in On-line and Off-line services. Usually, the information sharing by on-line and off-line services will be different. In this way, while the on-line services can access to the user information stored, the off-line services must collect this information independently. So it's obvious that the functionality of both could be different. In this work is developed a method to show users what are differences between on-line and off-line versions of the same service. Once showed this information, the user can select what version wants to use.

- Improve QoS of local services with external data. In previous works, has been exposed some method to store information inside smart-things by using tags, Bluetooth and others technologies. However, is very interesting for the user using this information to improve the capabilities of the device. In this work, will be presented a new technique based on IoT and smart-things to improve the capabilities of the physical activity recognition of users (L. M. Soria-Morillo, 2010). These systems allow the device to know many physical activities carried out by the user, but the main problem of them is the limited number of activities recognized. The most of these systems use accelerometers to detect pattern associated to some physical activity. 
- Improve QoS of remote process with local data. By storing the information on the own device, it's possible to share information between device and smart-thing. This information should be interesting for objects to improve de capabilities of them and theirs functionalities.

In the work presented along this paper, the main aim is to transforms the itemoriented view that Inter-net of Things has of the environment, towards a new concept in which the functionality is the cornerstone of the system. In this way, will be used a RESTful architecture to communicate the device (client-service) and the smart-thing access point (host-service). Later will be exposed the reasons why has been used this RESTful architecture for this purpose.

Furthermore, this change of view will facilitate the maintenance, scalability and developing of new services based on smart-things.

On the other hand, to share not only information but services with other users will let to get information and operate over remote objects thanks the access to its hostservices elements.

\subsection{Paper Structure}

Our paper is structured as follows. In Section 2, a scenario will be exposed to show the functionalities of the system, in which a real situation problem will be solved by using the techniques developed in this paper.

Section 3 presents the main ideas behind the Inter-net of Things concept and technologies used to develop our system.

Later, in Section 4, will be exposed an overview of the proposal, with different modules that compose the system. Along this section will be introduced the ways to share information between these modules as well as the structure that will have the information stored both the smart-thing server and user's mobile device.

In Section 5 is exposed in a more detailed way the different methods to execute the services. In this section will both be compared to determine limitations of each ones. Finally, will be presented a method allowing to users make a feedback about the service executed and based on users community feedbacks will be developed an recommendation system. This advice sys-tem allows to the user obtain automatically the best service for each item based on his/her information stored and his/her user profile.

Section 6 contains the different ways through which users can interact with smartthings. Initially, the differences between these methods will be where the user is and the kind of interaction between the user's device and the tagged object.

Finally, Section 7 contains conclusions which can be reached after the completion of this work.

\section{Scenario}

Mary really loves new technologies and therefore often she buys gadgets that provide new features to its environment and help her to carry out the daily activities. 
However, not everyone is happy at Mary's home. Daniel, her husband, seemed overwhelmed with the large number of devices in their home, so he generally made use of a few services, and is far from fully exploit all functionalities. But all that changed a few months ago with the arrival of the new detection and information sharing system.

The system, which provides the majority of devices at home of a recognition system based on NFC tags, make Mary and Daniel's mobile devices have become the main attraction of the house. In this way the system will be present at all times, inside and out-side the home.

Its 7:00 AM, the clock rings and Daniel is about to go at work. After picking up the phone from the table, our friend goes to the kitchen to drink a coffee cup made by him. To do this, Daniel bring his device close to the coffee maker, and immediately the device screen shows all functions offered by the machine (cleaning, make coffee, turn on, turn off, schedule, etc.). All these functions are obtained through the service interface provider by the manufacturer and this information is formatted by the mobile application. Our friend selects "make me my favorite coffee". Automatically, the service request information about Daniel's coffee tastes, which have been previously stored in the device. With this gesture, the coffee maker is able to make our friend's favorite coffee.

After taking the coffee, and automatically published this information through tweeter, Daniel is pre-pared to dress. To this end, the closet has a tag that, after being scanned, offers to Daniel different features, among which is "choosing clothes." Since Daniel device contains information about daily activities, the device sends to the service an indication that today is a business day and the time that Daniel chooses a formal costume. The suggested clothe based on weather expected for today is shown in Daniel's device.

After dressing and taking coffee, Daniel is ready to go at work, so down the stairs to the garage and ride in his car. At this time, due to localized services functionality, the option to open the garage door is displayed on the device. Once in the car, the device displays the option "search traffic incidences" to his work, allowing him to choose between an on-line application based on Google maps or activate the browser of his vehicle. Since the location of the work is stored on the device, the system automatically obtains all issues related to the route taken usually. Once checked that there is no traffic problems, starts his journey. Meanwhile in Facebook, the system automatically published that Daniel goes to work, and the status changed to "driving" warning to his friends that they should not call him.

The morning runs quietly while Mary, at home, prepares to listen to music. To do this action, Mary brings the device closer to the label on her Hi-Fi and chooses "default playlist". This option will allow to the Hi-Fi getting the most listened music in Mary's device and play at home stereo. However, Mary notes that the "play track" is a shared service provided by the system, and she publishes this service link among her social network. From this point, all her friends can control the music played in the Mary's Hi-Fi. Meanwhile, Daniel receives an alert on his device which indicates that Mary has shared a service with him. So without missing a beat, Daniel plays remotely the favorite song of both, a huge surprise for Mary. 


\section{Internet of Things Requirements}

Here should be explained on what is basis on the Internet of Things and what we need to know about the item (smart-thing) to get a working system. Also, will be described in what consist on the RESTful architecture and what is the advantage of sharing the information managed in this work through this technique, instead of using other systems such as SOAP. It will also include REST verbs, i.e. functions GET, POST, PUT and DELETE, and how can we modeling services by using these functions.

In this way, application installed on smartphones must link smart items with online services associated. So should be noted that if some change in original application were required, manufacturer just need to change the service implementation and the associated XML services description.

NFC device is responsible for storage the basic XML descriptive file, so interaction between user's smartphone and smart item is quick and straight. In fact, this not only allow wired services connected to manufacturer or OMS server, but execute preloaded servicer or applications directly with no internet connection needed. In the evaluation process by users this feature was very interesting for them, particularly when smart-items were in non-urban areas such as fields, farmhouses or cottage without internet coverage.

\section{Overall Process}

Overview of the execution process of an application based on smart-thing as provider and how the device exchanging necessary information with the application access point. The latter is very interesting from the point of view that the user mobile phone is the information manager, so all requested information from smart-thing services must be provided by the user's device. If some information is not stored, application controller will request it to the user.

Flowchart describing this section is shown in Figure 1. In this figure can be seen different subsystems identified in the main application, which will be responsible for manage applications and services pro-vides by smart-items.

First, identification subsystem is presented. Smartphone reads NFC tag and obtains all information contained on it, which describes services provided by smart items.

Then, action set is shown to user, such us smart-item interaction history, information stored into (useful for active items) or services provided. In the latter option, two alternatives can be offered: on-line or off-line services. If the service requires information about user, the user must approve information exchange. This feature is implemented as a security mechanism for deny non-approved information exchange, which is critical when contextual or personal information is consumed.

If required information is not storage or it can't provide by any context provider, system will require it to user. Depending of information type, a form will be displayed and user could fill the information called for.

Once all information is stored, service or local application could be executed and result will be shown to the user. 


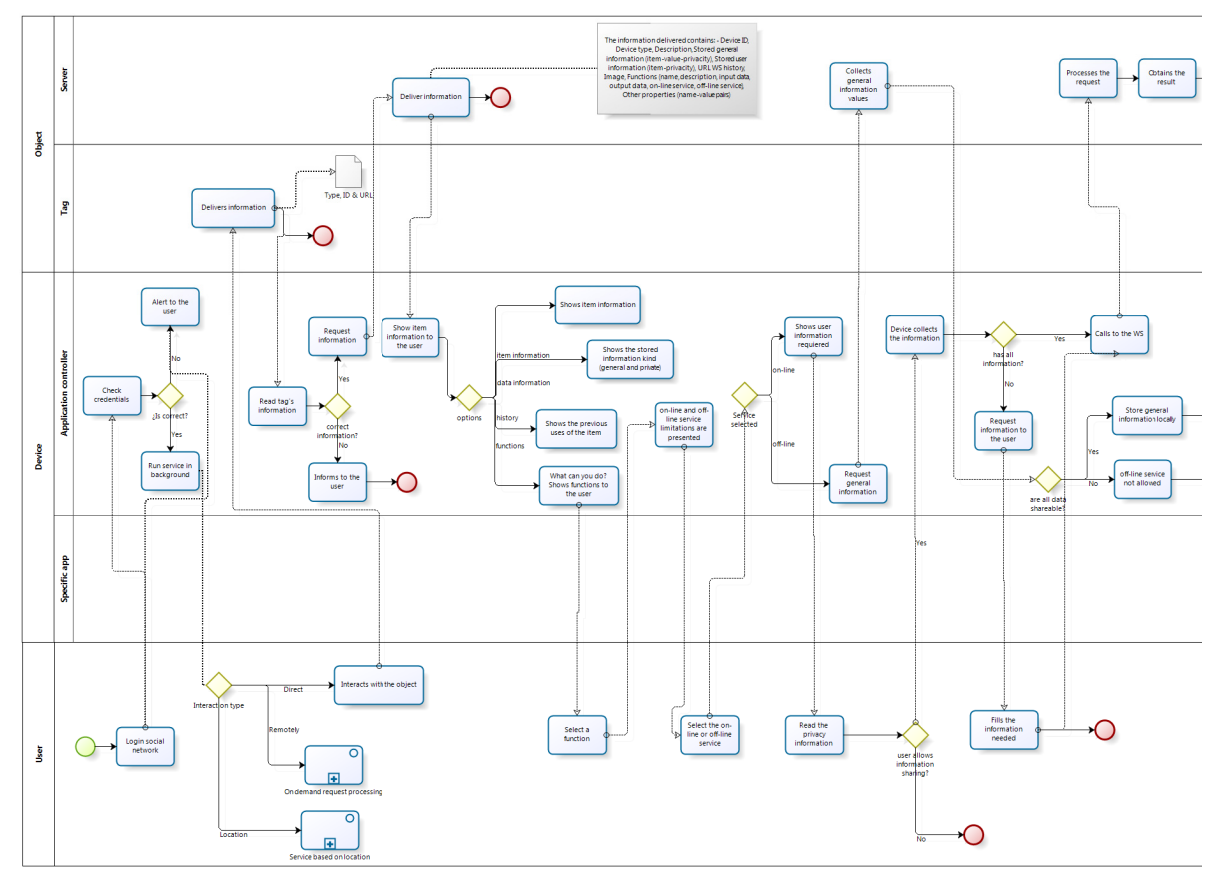

Fig. 1. Interaction flow diagram between users and Smart-Things

\section{$5 \quad$ Services}

Applications can provide some services to users for facilitate the use of smart-items or share information with them. In first place, must be distinguished two services kinds, on-line services implemented in the device and off-line services, implemented on the server. At this point we should explain what information is shown to the user to choose between on-line and off-line and, of course, what is the best method for interact with smart-items based on the environment.

For on-line services description, is needed to store a certain information kind to connect the application with a web service which provide the functionality. For this purpose has been defined a XML structure for describe de functionality and parameters required by this service.

The schema is composed by four big sections: item information block, services block, user interface configuration block and security assurance block. Now it will be described the content of previous blocks.

- Information block. Contains every kind of smart-thing static information, which could be understood as item specifications. This information is standardized into a specific ontology for allow queries and automatic information processing. Some information as location is provided in a dynamic way when objects are scanned by 
using NFC sensors embedded into smartphones. This means that must be needed to use two information block versions. First, a read-only version provided by OMS server, which contains common information for same type items. Second, a modified version of the previous document filled with concrete information of the labeled product, for instance, with product location or product owner.

- Services block. In this section is described the services offered by the product. Obviously, products can offers one or more services. Each service must contain general information about it, such as name, identification or description. At this point, must be noted that two kinds of services exist. On one hand, virtual service which results will be logic information, for instance, electronic program guide for some channel or temperature on the current city. On the other hand, real service could be implemented over multimedia objects. These services can change the real state for the object, such as change current program in a TV or change tuner on the radio. Regardless of the type of service, must be returned some value. A type set of values is defines in the structure for this purpose. In addition, often services need parameters for the execution. These parameters are defined in the structure and can be filled by the own user (basic input method) or obtained automatically from context providers (Luis M. Soria-Morillo, Juan A Ortega, 2011).

- User interface block. The aim of this section is to configure user interface appearance of different applications or services. Appearance scopes are not deeps and it just allows customizing concrete aspects. This decision was taken for achieve standard interfaces to make easier human-application interactions.

- Security assurance block. Due to it's possible to define location based services, which are activated when users are in a particular place. To avoid intrusions on personal systems, users can define services that just can be executed by a subset of users. For instance, it could be useful for allow TV tuning. If some neighbor is in our house, may not be interesting he/she can change the TV channel, but yes for our child. Furthermore, NFC technology provides the possibility of implementing a hardware security system by restricting the number of de-vices that can read a particular tag (Haselsteiner \& Breitfuß, 2006).

\subsection{Off-Line Services}

These services are implemented like typical applications. These applications will be provided by the own smart-thing and could be installed in a transparent way in the user mobile phone just reading the associated tag. These applications can't access to the information stored on the device, due to could be break the security of this information.

\subsection{On-Line Services}

These services give to the user a new way to inter-act with smart-thing. Smart-things in this case, not only will provide information to the user, but will allow carrying 
out some processing. These processes are so called "services", and it's the main difference between passive objects and active objects. First ones only allow request information about the state, while the active objects allow to the user interact with them and request that some service (process) was made.

\section{Interaction Methods}

An interaction method is considered as a way in which a user can interact with his/her environment services. At first there are three ways of interaction, direct interaction and remote interaction and interaction by location.

- A direct interaction is presented when users maintain physical contact with items. This is the most trivial interaction and it's used most of the time nowadays.

- If the previous physical interaction is replaced by any smartphone with some application to control the smart-item we are facing to a re-mote interaction. This interaction kind allows delivering information needed by the function by querying the user preferences.

- Interaction by location is a new kind of inter-action system that allows publishing functionalities about smart-items just on a concrete location. It's to say, if some user is in the kitchen and a certain smart-item only offers some functionality in the living room, it won't be available where the user is.

\section{Conclusions}

This paper establishes the basis of a new system which will be able to share information in an automatic way between smart-items and user's smartphone. Furthermore, thanks to the proposal ontology, every item could be described by a set of properties which will be used for apply filters over the items and by this way, assigns a set of authorizations for share just some information about the user, if it is required by the application.

The proposed system will allow interacting with smart-items across a new concept: digital me. In this structure, whole information about user is stored in his/her smartphone, being that needed inputs for functions offered by items. Considering this concept, user does not have to introduce the needed information, but that will be obtained in an automatic way.

Obviously this is not a trivial approach, due to the quantity and kinds of scenarios are wild. For this purpose and to lead the way of the new system, a concrete scenario has been raised.

Acknowledgements. This research is supported by the Spanish Ministry of Science and Innovation R\&D project ARTEMISA (TIN2009-14378-C02-01). 


\section{References}

1. Alexander Kröner, A.J.: Augmenting Cognition With a Digital Episodic Memory, Künstliche Intelligenz, KI, pp. 51-57 (2008)

2. Andreas Björklind, S.H.: Ambient Intelligence to Go. Research Report SAR-03-03 (2003)

3. Boris Brandherm, J.H.: Demo: Authorized access on and interaction with Digital Product Memories. In: Eight Annual IEEE International Conference on Pervasive Computing and Communications, PerCom 2010, pp. 838-840. IEEE, Mannheim (2010)

4. von Reischach, F., Michahelles, F., Guinard, D., Adelmann, R., Fleisch, E., Schmidt, A.: An Evaluation of Product Identification Techniques for Mobile Phones. In: Gross, T., Gulliksen, J., Kotzé, P., Oestreicher, L., Palanque, P., Prates, R.O., Winckler, M. (eds.) INTERACT 2009. LNCS, vol. 5726, pp. 804-816. Springer, Heidelberg (2009)

5. Kranz, M., Holleis, P., Schmidt, A.: Embedded Interaction: Interacting with the Internet of Things. IEEE Internet Computing, 46-53 (2010)

6. Soria-Morillo, L.M.,, J.A.-G.-A.: Tracking system based on accelerometry for users with restricted physical activity. In: 23rd International Conference on Industrial Engineering and Others Applications of Applied Intelligent Systems, iea/aie 2010, Córdoba, Spain (2010)

7. Lacmanovic, I., Radulovic, B., Lacmanovic, D.: Contactless payment systems based on RFID technology. In: 2010 Proceedings of the 33rd International Convention MIPRO, pp. 1114-1119. IEEE Computer Society, Opatija (2010)

8. Ondrus, J.P.: An Assessment of NFC for Future Mobile Payment Systems. In: Conference on the Management of Mobile Business, p. 43. IEEE Computer Society, Washington, DC (2007)

9. Roy, W.,, F.K.: Bridging physical and virtual worlds with electronic tags. In: CHI 1999: Proceedings of the SIGCHI Conference on Human Factors in Computing Systems, pp. 370-377. ACM, New York (1999) 